\title{
Performance Analysis of Integrative QoS Scheme for Routers
}

\author{
Jih-Hsin Ho \\ Department of Leisure \& Information Management, Taiwan Shoufu University, Tainan City72153, Taiwan
}

\begin{abstract}
This paper proposes an integrative scheme of DiffServ (differentiated service) for the IP-based network. In the scheme, QoS (Quality of Service) is ranked according to the level of both the queuing delay and the drop precedence that base on the relative differentiated service; it does not take the resource reservation problem into consideration for making its implementation more simple and flexible. We will propose an implemented architecture including edge routers and core routers, and the PHB (per-hop-behavior) architecture of the routers is generalized. We adopt ERED (extended random early discard) mechanism for drop precedence and use proportional delay differentiation for queuing delay. In the proportional delay differentiation topic, we propose a new WTP (waiting time priority)-like algorithm. Besides, a new stochastic petri net model of the PHB model is given, and the performance of whole implemented PHB architecture is analyzed, too.
\end{abstract}

Key words: QoS, PHB, WTP.

\section{Introduction}

So far there are two approaches for achieving end-to-end QoS (quality of service) [1] in IP-based network: IntServ (integrated service) [2] and DiffServ (differentiated service) [3], where IntServ is more complex and less scalable than DiffServ. Over the past years, there has been an amount of research studying in the field of DiffServ field that is worth studying.

Today's Internet comprises of multiple interconnected domains. Each domain is constructed by core routers in the insider, and through edge (boundary) node that include ingress router and egress router. A DiffServ domain scenario is shown as in Fig. 1. Before entering a DiffServ domain, the different data flows are aggregated [4] by core routers process packets only based on PHBs that encoded in DSCP of the packet header. Currently, the IETF (internet engineering task force) has defined one class for EF

Corresponding author: Jih-Hsin Ho, Ph.D., assistant professor, research fields: performance evaluation, WDM networks, Internet, QoS. E-mail: hjsin@tsu.edu.tw. (expedited forwarding) [5] and four classes for AF (assured forwarding) [6].

AF is implemented as AS (assured service) [6]. Originally, it was proposed to use the RIO (red-in/out) approach to ensure the "expected capacity" specified by traffic profile. The basic idea is, upon each packet arrival, if the traffic rate is within the traffic profile, the packet is marked as "in", otherwise, it is marked as "out". In the DiffServ, core router is preferentially drops the packets that are marked as "out" during network congestion. Currently, the AF PHB [6] defined by IETF specifies four traffic classes with three-drop precedence levels (or three colors) within each class. There are 12 DSCPs for AF class. Within an AF class, there are three colors to choice to mark as green, yellow, and red, where green has the lowest drop probability and red has the highest drop probability. The mark technology is proposed by Wang, F. G. in Ref. [7], "random early demotion and promotion marker for assured service", it is expected that the scheme can be used for supporting differentiated services through an efficient marking process. 


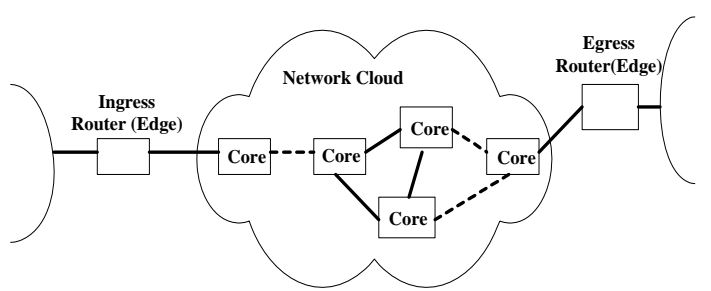

Fig. 1 DiffServ domain scenario.

In this paper we will propose an implemented architecture including the edge routers and core routers. In edge router and core router PHB architecture will be generalized. We will adopt ERED (extended random early discard) mechanism for the drop precedence level and use proportional delay differentiation for queuing delay. In the proportional delay differentiation, we propose a new WTP (waiting time priority)-like algorithm, and an stochastic Petri Net models of the PHB model. The performance of the whole PHB implemented architecture is analyzed, too.

This paper is organized as follows: Section 2 proposed the integrative scheme of DiffServ, together with the implemented architecture. An stochastic Petri Net models of PHB model is constructed in Section 3. The performance analysis of the model is discussed in Section 4; finally a conclusion is given in Section 5 .

\section{Implemented Architecture}

In this section, an integrative scheme of differentiated services will be presented, in which DiffServ implemented architecture divided into two modules: DS (DiffServ) classifier module and DS queuing discipline module. The DS implemented architecture is shown in Fig. 2. Whole DiffServ domain scenario has been discussed in the above chapter. Within this domain, the edge routers contain two modules but the core routers only contain DS queuing discipline module.

\section{- DS Classifier Module}

This module is responsible to classify any incoming data flows or data packets in the edge routers to find the corresponding traffic profiles with a DSCP for them.

The DS classifier module classifies a data packet using the parameters of burst rate and burst size derived

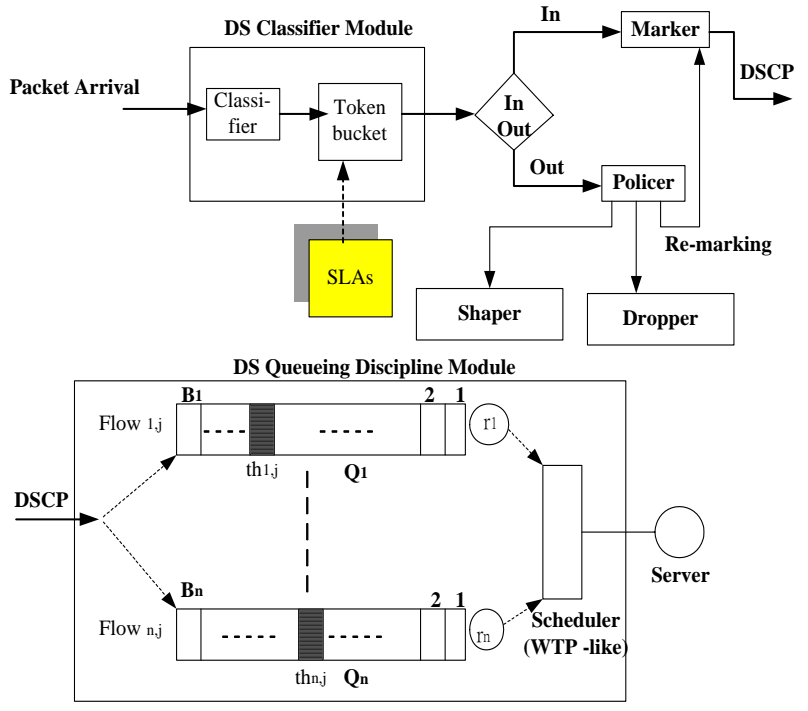

Fig. 2 DS implemented architecture.

from the packet's characteristic corresponding the SLA (service level agreement) or profile-list through a token bucket mechanism. If the packet's traffic rate is within the SLA, the packet is called "in-of-profile"; otherwise, it is called "out-of-profile". The in-of-profile packets are marked with DSCP bits in the packet's IP header, and the packets are transmitted to the DS queuing discipline module. The out-of-profile packets are handled by policer. The policer is enforced via some functions consisting of dropper, shaper, degrading and remark. We discard noncompliant packets by dropper function, holding back out-of-profile packets until they conform to the specified traffic profile by shaper function, and combining with policing in and out-of-profile packets are marked with different DSCP by degrading and remark functions.

\section{- DS Queuing Discipline Module}

DS queuing discipline module is named PHB module, too. A more general AF PHB will be introduced. In Fig. 2, there are $n$ queues, and they are numbered with the priorities of queuing delay level. Let $Q_{i}$ denote the queue with level $i$, whose buffer size is $B_{i}$. The $Q_{1}$ has highest scheduling priority and $Q_{n}$ has the lowest scheduling priority. In each $Q_{i}$, there are several drop precedence levels ranking $j$. The ranking $j+1$ has lower loss precedence than ranking $j$. Let $\mathrm{Flow}_{i, j}$ denote the traffic aggregate with queuing 
delay level $i$ and drop precedence ranking $j$.

The AF PHB in RFC 2597 defined by IETF specifies four queuing delay levels $(i=4)$ with three drop precedence ranking $(j=3)$ with each level. In all, there are 12 DSCP for AF PHB. Based on the assumption of ERED mechanism on drop precedence and using proportional delay differentiation on queuing delay, we obtain expressions of performance analysis that characterize the service to provide different kinds of packets. Our analysis is based on AF PHB (12 DSCP) and it can be easily extended to generalize assured forwarding. We next describe the mechanisms for drop precedence level and queuing delay in detail.

\section{- Drop Precedence Ranking}

A simplified model of assured forwarding is classified into two drop precedence ranking ("IN" an "OUT") at the edge routers, depending on whether the data flow follows the SLA or not. When congestion occurs, OUT packets are discarded with a higher probability than IN packets. This operation is usually implemented by a RIO queue management scheme, which typically applies both IN and OUT packets.

Within an AF class, a packet is marked as one of three colors - green, yellow, and red—where green has the lowest drop probability and red has the highest drop probability. Here we use ERED (extended RED) for service differentiated of different colors. ERED scheme has three parameters: minimum threshold, maximum threshold and drop probability for three-color packets. Formally, if the queue length is $n$ at the arrival of a packet, the probability of the packet to be accepted into the queue is

$$
\alpha(n, Q i)=p_{G} \alpha^{G}(n, Q i)+p_{Y} \alpha^{Y}(n, Q i)+p_{R} \alpha^{R}(n, Q i)
$$

where, $p_{G}$ is the probability that a packet is an green packet, $p_{Y}$ is the probability that a packet is an yellow packet and $p_{R}$ is the probability that a packet is an red packet.

$$
p_{G}+p_{Y}+p_{R}=1
$$

where, $\alpha^{G}(n, Q i), \alpha^{Y}(n, Q i)$ and $\alpha^{R}(n, Q i)$ respectively are the probabilities of an green, yellow and red packet to be accepted into the queue Qi. Base on the properties of ERED, we have

$$
\left[\begin{array}{l}
\alpha^{G}(n, Q i)=1, n \leq \min ^{G} \\
\alpha^{Y}(n, Q i)=1, n \leq \min ^{Y} \\
\alpha^{R}(n, Q i)=1, n \leq \min ^{R} \\
\alpha^{G}(n, Q i)=0, \max ^{G}<n \\
\alpha^{Y}(n, Q i)=0, \min ^{Y}<n \\
\alpha^{R}(n, Q i)=0, \min ^{R}<n \\
\alpha^{G}(n, Q i)=1-\frac{p^{G}\left(n-\min ^{G}\right)}{\max ^{G}-\min ^{G}}, \min ^{G}<n<\max ^{G} \\
\alpha^{Y}(n, Q i)=1-\frac{p^{Y}\left(n-\min ^{Y}\right)}{\max ^{Y}-\min ^{Y}}, \min ^{Y}<n<\max ^{Y} \\
\alpha^{R}(n, Q i)=1-\frac{p^{R}\left(n-\min ^{R}\right)}{\max ^{R}-\min ^{R}}, \min ^{R}<n<\max ^{R}
\end{array}\right]
$$

\section{- Queuing Delay Level}

There are several queuing delay mechanisms that can be used such as PQ (priority queue), WFQ (weighted fair queuing), and QLT (queue length threshold), etc.. In this paper we adopt the proportional delay differentiation service model.

The proportional delay differentiation service model first is proposed in Refs. [8, 9] by Dovrolis et al.. The model has two objectives. First, it should provide consistent service differentiation between classes. Second, it should allow the quality spacing by network operator. So this model has advantages of predictable and controllable in the differentiated service. Dovrolis [9] proposed an algorithm called the WTP (waiting-time priority) scheduler, which is based on Kleinrock's [10] time-dependent priority algorithm. In spite of WTP algorithm can approach the proportional delay differentiation between classes, it is not scalable due to computation complexity during packet forwarding. We will propose an improved algorithm called WTP-like algorithm in the following discussions.

We suppose that each router has a pre-specified number of delay classes $(\mathrm{N}=4)$ and provides a set of control variables $r_{i}$, where $r_{4}<r_{3}<r_{2}<r_{1}$, queues of different delay classes are served such that the average delay experienced by packets in a delay class is inversely proportional to the control variable. That is

$$
\frac{\overline{d_{i}}(t)}{\overline{d_{j}}(t)}=\frac{r_{j}}{r_{i}}
$$


In other word, if $\overline{d_{i}}(t)$ is the average delay for class $i$ at time $t$, then the goal is to achieve

$$
\left|\overline{d_{i}}(t) r_{i}-\overline{d_{j}}(t) r_{j}\right| \rightarrow 0
$$

In the WTP scheduler, the priority of a packet increases proportionally with its waiting time. Hence, when a router needs to select a packet for transmission at time $t$, it selects a queue $j$ for transmission such that

$$
j \leftarrow \operatorname{argmax}\left\{w_{i}(t) \times r_{i}\right\}
$$

In our scheduler called WTP-like scheduler, the priority of packet increases proportionally with its delay time. The delay time at time $t d_{i}(t)$ which is transferred another formula by Little's theorem and Eq. (4) as shown in Eq. (7), where $B_{i}(t)$ is queue size and $\lambda_{i}(t)$ is arrival rate for each queue $i$.

$$
d_{i}(t)=B_{i}(t) / \lambda_{i}(t)=k / r_{i}
$$

We improved the WTP scheduling reducing the computation of average waiting time instead of average delay time. The implementation for WTP-like algorithm is shown in Fig. 3. In De_queue procedure, it selects a queue $j$ for transmission such that

$$
j \leftarrow \underset{\operatorname{argmax}}{i}\left\{B_{i}(t) \cdot r_{i} / \lambda_{i}(t)\right\}
$$

\section{Stochastic Petri Net Models}

Generally speaking, the DS queuing discipline module (AF PHB) can be modeled with SPN (stochastic petri net) models. We further review the SPN models as it is proposed in Ref. [11]. The SPN models have places $P$, transitions $T$, input and output $\operatorname{arcs} A$, an initial marking $M$ and the set of average transition rates $\lambda$ defined as $S P N \cong(P, T, A, M, \lambda)$. The SPN models follow an theorem as for any finite place, finite transition, marked SPN is isomorphic to a one-dimensional discrete space Markov process.

En_queue Procedure ( )

Based on some basic knowledge on SPN, Fig. 4 shows the SPN model of DS queuing discipline module. The transitions and the places in Fig. 4 are described as follows:

Qi: represents the buffer queue of queuing delay level $i$, whose capacity is $B i$;

$$
\left\{B_{i}(t) \leftarrow B_{i}(t)+1\right\} ; \begin{aligned}
& * \\
& \text { class } * /
\end{aligned}
$$

De_queue Procedure ( )

$$
\left\{j=\max _{i}\left\{B_{i}(t) r_{i} / \lambda_{i}(t)\right\} ;\right.
$$

transmit from delay class $j$; $/ *$ select a packet to transmit from a queue */

$\left.B_{j}(t) \leftarrow B_{j}(t)-1 ;\right\}$

Fig. 3 WTP-like algorithm.

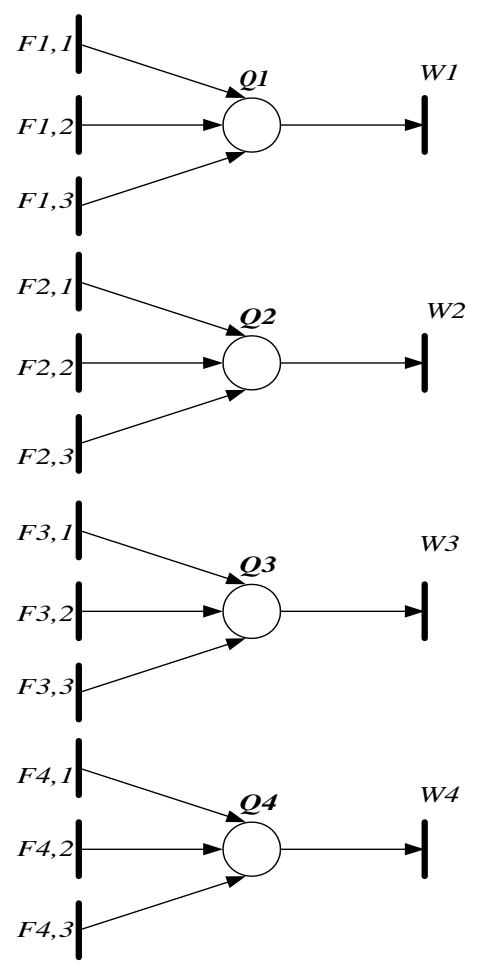

Fig. 4 The stochastic petri net model of AF PHB.

$F i, j$ : represents the arrival of packets of Flowi,j with queuing delay level $i(1 \leq i \leq 4)$ and drop precedence ranking $j(1 \leq j \leq 3)$ with mapping to red, yellow and green. Its firing rate is $\lambda^{j}(Q i)$;

$W i$ : represents the server of queue $i$, whose service rate is $\mu_{i}$. Here we assume that packets with same drop precedence ranking are served with the same rate.

In the SPN model, the probability associated with transition $F i, j$ is $\sigma i, j$, so that the actually firing rate of $F i, j$ is $\sigma i, j \times \lambda^{j}(Q i)$. And the probability associated with transition $W i$ is $\xi i$, so that the actually firing rate of $W i$ is $\xi i \times \mu_{i}$. Here $\sigma i, j$ describes the ERED drop control policy in the condition of Eq. (3). And $\xi i$ describes the 
proportional delay differentiation service, which refers to WTP-like algorithm as

$$
\xi_{i}\left(Q_{i}\right)=\left\{\begin{array}{l}
1, i \leftarrow \arg \max _{i}\left\{B_{i}(t) \cdot r_{i} / \lambda_{i}(t)\right\} \\
0, \text { otherwise }
\end{array}\right.
$$

\section{Performance Analysis}

Based on the SPN of AF PHB in Fig. 4, the number of packet in the four queues is a Markov process with state space $\boldsymbol{S}=\left\{\left(0, Q_{1}\right),\left(1, Q_{1}\right), \ldots \ldots,\left(K, Q_{1}\right),(0\right.$, $\left.Q_{2}\right),\left(1, Q_{2}\right) \ldots \ldots,\left(K, Q_{2}\right),\left(0, Q_{3}\right),\left(1, Q_{3}\right) \ldots \ldots,\left(K, Q_{3}\right),(0$, $\left.\left.Q_{4}\right),\left(1, Q_{4}\right) \ldots \ldots,\left(K, Q_{4}\right)\right\}$, where $0,1,2, \ldots ., K$ denote the queue length and $K$ is the size of the queue. The above Markov chain with large state $(|S|=4(K+1))$ and multiple dimensions is a difficult problem. One of the possible ways accepts an approximate approach based on the analysis with one-dimension models. Since the SPN model in Fig. 4 has been refined, each structural independent part is a submodel.

The state transition diagram of approximate approach is shown in Fig. 5.

Since an ERED queue management scheme is assumed in our model. Some packets will be dropped and do not influence the state of the four queues. In ERED, when the queue length is $n$, the actual arrival rate of Green packets, yellow packets, red packets and all packets to four queues are

$$
\begin{aligned}
& \lambda^{G}(n, Q i)=\lambda_{Q i} \alpha^{G}(n, Q i) \\
& \lambda^{Y}(n, Q i)=\lambda_{Q i} \alpha^{Y}(n, Q i) \\
& \lambda^{R}(n, Q i)=\lambda_{Q i} \alpha^{R}(n, Q i) \\
& \lambda(n, Q i)=\lambda_{Q i} \alpha(n, Q i)
\end{aligned}
$$

where, we assume the packets arrive in the four queues according to a Poisson process with rate $\lambda_{\mathrm{Q} i}$ respectively. $\alpha G(n, Q i), \alpha Y(n, Q i)$ and $\alpha R(n, Q i)$ are given by Eq. (3). Then, when the queue length is $n$, the actual packet arrival rate (i.e., arrivals which will not be dropped before entering the four queues) is

$$
\lambda_{n, Q i}=p_{G} \lambda^{G}(n, Q i)+p_{Y} \lambda^{Y}(n, Q i)+p_{R} \lambda^{R}(n, Q i)
$$

One challenge in our analysis is the relationships among submodels, i.e., the relations among submodels have been described in the predicates of transition $\mathrm{Wi}$ that involves the mean service rate. Thus, we must solve the mean service rate $\mu i$ in steady (statistical-equilibrium) state.

The steady-state distribution, $\pi(s), \quad s \in S$, is determined by the following balance equations:

$$
\vec{\pi}(s) A=0, \sum \vec{\pi}(s)=1
$$

where,

$$
\begin{aligned}
& \vec{\pi}(s)=[\pi(0, Q i), \pi(1, Q i), \pi(2, Q i), \ldots, \pi(K, Q i)] \\
& A=\left[\begin{array}{cccccc}
-\lambda_{Q Q i} & \lambda_{Q, Q i} & 0 & \cdot & \cdot & 0 \\
\mu & -\left(\lambda_{1, Q i}+\mu\right) & \lambda_{1, i} & 0 & . & 0 \\
\cdot & \cdot & \cdot & \cdot & \cdot & \cdot \\
0 & \cdot & \cdot & \mu & -\left(\lambda_{K-1, i}+\mu\right) & \lambda_{K-1, i} \\
0 & 0 & 0 & 0 & \mu & -\mu
\end{array}\right]_{K+1)(K+1)}
\end{aligned}
$$

Let $\operatorname{loss}_{\mathrm{Qi}}^{\mathrm{G}}, \operatorname{loss}_{\mathrm{Qi}}^{\mathrm{Y}}$ and $\operatorname{loss}_{{ }_{\mathrm{Qi}}}^{\mathrm{R}}$ be the drop probability for green packets, yellow packets and red packets in each Qi, respectively. Using the PASTA (Poisson arrival see time averages) property [12], we have:

$$
\begin{aligned}
& \operatorname{loss} \underset{Q i}{G}=1-\sum_{n=0}^{K} \alpha^{G}(n, Q i) \pi(n, Q i) \\
& \operatorname{loss} \underset{Q i}{Y}=1-\sum_{n=0}^{K} \alpha^{Y}(n, Q i) \pi(n, Q i) \\
& \operatorname{loss}{\underset{Q i}{R}}^{Y}=1-\sum_{n=0}^{K} \alpha^{R}(n, Q i) \pi(n, Q i)
\end{aligned}
$$

We can also get the effective throughput of packets as:

$$
\begin{aligned}
& \text { eff }{ }_{Q i}^{G}=\lambda_{Q i} p^{G} \sum_{n=0}^{K} \alpha^{G}(n, Q i) \pi(n, Q i) \\
& \text { eff }{ }_{Q i}^{Y}=\lambda_{Q i} p^{Y} \sum_{n=0}^{K} \alpha^{Y}(n, Q i) \pi(n, Q i) \\
& \text { eff }{ }_{Q i}^{G}=\lambda_{Q i} p^{R} \sum_{n=0}^{K} \alpha^{R}(n, Q i) \pi(n, Q i) \\
& \text { eff }{ }_{Q i}=\lambda_{Q i} \sum_{n=0}^{K} \alpha(n, Q i) \pi(n, Q i)
\end{aligned}
$$

Applying Little's theorem, we have the mean delay of the queue $Q i(1 \leq i \leq 4)$, respectively

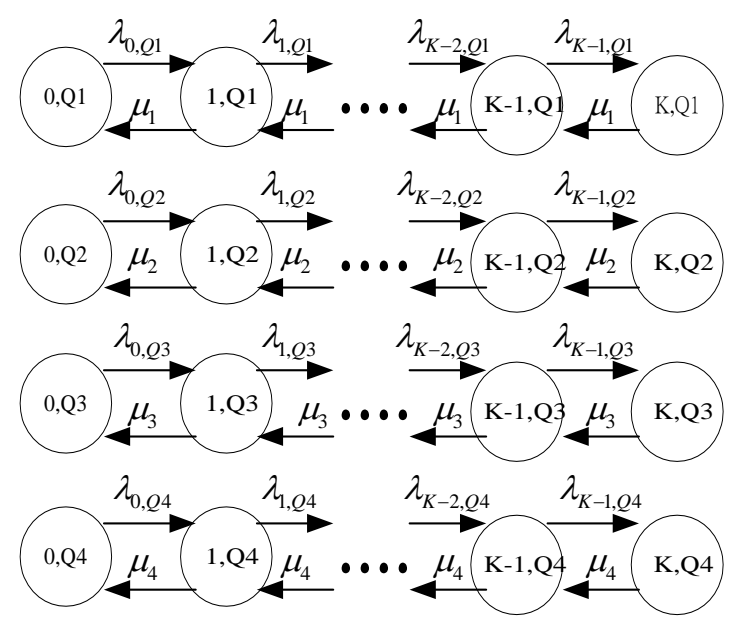

Fig. 5 The state transition diagram of approximate approach. 


$$
E(d i)=\sum_{n=0}^{K} n \pi(n, Q i) / e f f_{Q i}
$$

\section{Conclusions}

In this paper, we have presented an integrative scheme of differentiated service for the IP-based network. The scheme takes the drop precedence ranking and the queuing delay level into consideration. In queuing delay mechanism, we have proposed a WTP-like algorithm to reduce the computation of average waiting time instead of average delay time. The ERED is the queue management policy. Based on stochastic Petri Net model, we obtained many approximate approach solutions such as loss probability, effective throughput and mean delay of the queue. Only how to obtain the mean service rate in each queue need to be further studied.

Our analysis is based on AF PHB (12 classes) in RFC 2597 and it can be easily extended to general PHB (more than 12 classes). Future work includes simulation of our scheme under both Poisson and self-similar arrivals and modeling of arrival traffic for both TCP and UDP.

\section{References}

[1] McWherter, D.T., Sevy, J., and Regli, W.C. 2000. "Building an IP Network Quality-of-Service Testbed." IEEE Internet Computing 4 (4): 65-73.

[2] Zhang, L., Berson, S., Herzog, S., and Jamin, S. 1997. "Resource ReserVation protocol (RSVP)—Version 1 functional specification.” RFC 2205, United States.

[3] Blake, S., Black, D., Carlson, M., Davies, E., Wang, Z., and Weiss, W. 1998. "An Architecture for Differentiated Services”, IETF RFC 2457, http://www.rfc-editor.org/rfc/rfc2475.txt.

[4] Schmitt, J. Karsten, M., Wolf, L., and Steinmetz, R. 1999. "Aggregation of Guaranteed Service Flows." In Proceedings of the Seventh International Workshop on Quality of Service, 147-55.

[5] Dovrolis, C. etc. 1999. “A case V. Jacobson etc., “ An Expedited Forwarding PHB ”, IETF RFC 2598, June 1999. http: // www. rfc-editor.org/ rfc/rfc2598.

[6] Forwarding PHB Group, “IETF RFC 259 7”, June 1999. http: //www. rfc-editor.org/ rfc/rfc 2597.

[7] Wang, F. G., Mohapatra, P., Mukherjee, S., Bushmitch, D. 2000. "A Random Early Demotion and Promotion Marker for Assured Services.” IEEE Journal of Selected Areas in Communications 18 (12): 2640-50.

[8] Dovrolis, C. and Ramanathan, P. 1999. "A Case for Relative Differentiated Services and the Proportional Differentiation Model.” IEEE Network: The Magazine of Global Internetworking 13 (5): 26-34.

[9] Dovrolis, C., Stiliadis, D., and Ramanathan, P. 1999. "Proportional Differentiated Services: Delay Differentiation and Packet Scheduling.” ACM SIGCOMM Computer Communication Review 29 (4): 109-20.

[10] Kleinrock, L., “Priority Queueing”, Queueing System II.

[11] Molloy, M. K. 1982. "Performance Analysis Using Stochastic Petri Nets.” IEEE Transactions on Computer C-31 (9): 913-7.

[12] May, M., Bolot, J. C., Jean-Marie, A., and Diot, C. 1999. "Simple Performance Models of Differentiated Services Scheme for Internet." In Proceedings of Eighteenth Annual Joint Conference of the IEEE Computer and Communications Societies, 1385-94. 